\title{
ANSWER TO A QUESTION OF KOLMOGOROV
}

\author{
RICHÁRD BALKA, MÁRTON ELEKES, AND ANDRÁS MÁTHÉ \\ (Communicated by Tatiana Toro)
}

\begin{abstract}
More than 80 years ago Kolmogorov asked the following question. Let $E \subseteq \mathbb{R}^{2}$ be a measurable set with $\lambda^{2}(E)<\infty$, where $\lambda^{2}$ denotes the twodimensional Lebesgue measure. Does there exist for every $\varepsilon>0$ a contraction $f: E \rightarrow \mathbb{R}^{2}$ such that $\lambda^{2}(f(E)) \geq \lambda^{2}(E)-\varepsilon$ and $f(E)$ is a polygon? We answer this question in the negative by constructing a bounded, simply connected open counterexample. Our construction can easily be modified to yield an analogous result in higher dimensions.
\end{abstract}

\section{INTRODUCTION}

The following question was posed by M. Laczkovich in [4]. Let $\lambda^{d}$ stand for the $d$-dimensional Lebesgue measure.

Question 1.1 (Laczkovich). Let $E \subseteq \mathbb{R}^{d}(d \geq 2)$ be a measurable set such that $\lambda^{d}(E)>0$. Does there exist a Lipschitz onto map $f: E \rightarrow[0,1]^{d}$ ?

For $d=2$ the positive answer to Question 1.1 follows from a result of N. X. Uy [6], and D. Preiss also solved this partial problem by completely different methods. J. Matoušek [5] proved the following stronger, 'absolute constant' version based on a well-known combinatorial lemma due to Erdős and Szekeres. (For the definition of 1-Lipschitz map see the Preliminaries section.)

Theorem 1.2 (Matoušek). There exists a constant $c>0$ such that for any measurable set $E \subseteq \mathbb{R}^{2}$ with $\lambda^{2}(E)=1$ there exists a 1-Lipschitz onto map $f: E \rightarrow[0, c]^{2}$.

Question 1.1 is still open for dimensions $d>2$. Theorem 1.2 states that we can contract every set of the plane with positive measure onto a square such that it 'does not lose too much from its measure'. Can we do this so that the loss of the measure is arbitrarily small? It is easy to see that this is not possible if we require the range to be a square, but how about polygons? Note that by polygons we mean a wider class of objects than its standard definition does:

Definition 1.3. We say that $P \subseteq \mathbb{R}^{2}$ is a polygon if $\partial P$ can be covered by finitely many line segments.

Received by the editors February 1, 2013 and, in revised form, October 16, 2013.

2010 Mathematics Subject Classification. Primary 28A75, 26A16.

Key words and phrases. Lebesgue measure, Lipschitz map, contraction, polygon.

The authors gratefully acknowledge the support of the Hungarian Scientific Research Fund grants no. 72655 and 104178.

The second author was supported by the Hungarian Scientific Research Fund grant no. 83726.

The third author was supported by the Leverhulme Trust. 
The next question is due to A. N. Kolmogorov; it was quoted by P. Alexandroff in a letter written to F. Hausdorff (see [1] and [2]).

Question 1.4 (Kolmogorov). Let $E \subseteq \mathbb{R}^{2}$ be a measurable set with $\lambda^{2}(E)<\infty$, and let $\varepsilon>0$. Does there exist a contraction $f: E \rightarrow \mathbb{R}^{2}$ such that $\lambda^{2}(f(E)) \geq$ $\lambda^{2}(E)-\varepsilon$ and $f(E)$ is a polygon?

The main goal of the paper is to answer Question 1.4 in the negative.

Theorem 1.5. There exist a bounded, simply connected open set $U \subseteq \mathbb{R}^{2}$ and $\varepsilon>0$ such that if $f: U \rightarrow \mathbb{R}^{2}$ is a contraction with $\lambda^{2}(f(U)) \geq \lambda^{2}(U)-\varepsilon$, then $f(U)$ is not a polygon.

In contrast to Question 1.1 the higher-dimensional versions of Question 1.4 are not more difficult than the original one. The analogue of Theorem 1.5 can be proved similarly for every dimension $d>2$ with straightforward modifications.

The structure of the paper is as follows. In Section 2 we recall some notation and definitions which we use in this paper. In Section 3 we prove Theorem 1.5 . Finally, in Section 4 we collect the open problems.

\section{Preliminaries}

Let $B(x, r)$ stand for the closed ball of radius $r$ centered at $x$. For a set $A \subseteq \mathbb{R}^{2}$ we denote by $\operatorname{int} A, \operatorname{cl} A$ and $\partial A$ the interior, closure and boundary of $A$, respectively. A function $f: A \rightarrow \mathbb{R}^{2}$ is said to be Lipschitz if there exists a constant $c \in \mathbb{R}$ such that $|f(x)-f(y)| \leq c|x-y|$ for all $x, y \in A$. The smallest such constant $c$ is called the Lipschitz constant of $f$ and denoted by $\operatorname{Lip}(f)$. If $\operatorname{Lip}(f) \leq 1$, then $f$ is a 1-Lipschitz map, and if $\operatorname{Lip}(f)<1$, then $f$ is a contraction. A function $f: A \rightarrow \mathbb{R}^{2}$ is called an isometry if $|f(x)-f(y)|=|x-y|$ for all $x, y \in A$, and $f$ is a local isometry if every point of $A$ has a neighborhood $U$ such that $\left.f\right|_{U \cap A}$ is an isometry. If $A, B \subseteq \mathbb{R}^{2}$, then let $\operatorname{dist}(A, B)=\inf \{|x-y|: x \in A, y \in B\}$.

For the sake of simplicity, let $\lambda=\lambda^{2}$ stand for the two-dimensional Lebesgue measure.

\section{THE PROOF}

First we need the following lemma.

Lemma 3.1. Assume that $U \subseteq \mathbb{R}^{2}$ is a non-empty, bounded, connected open set and $f: U \rightarrow \mathbb{R}^{2}$ is a 1-Lipschitz map such that $\lambda(f(U))=\lambda(U)$. Then $f$ is the restriction of an (affine) isometry $\psi: \mathbb{R}^{2} \rightarrow \mathbb{R}^{2}$.

Proof. Recall that if the set $A \subseteq \mathbb{R}^{2}$ is not collinear and $g: A \rightarrow \mathbb{R}^{2}$ is an isometry, then there exists a unique affine isometry $\psi: \mathbb{R}^{2} \rightarrow \mathbb{R}^{2}$ such that $g=\left.\psi\right|_{A}$. Indeed, let $S \subseteq A$ be a non-degenerate triangle; then it is easy to see that there exists a unique affine isometry $\psi: \mathbb{R}^{2} \rightarrow \mathbb{R}^{2}$ such that $\left.g\right|_{S}=\left.\psi\right|_{S}$. Assume to the contrary that there is an $x \in A$ such that $g(x) \neq \psi(x)$. Then $x \neq \psi^{-1} g(x)$, and for all $s \in S$

$$
|s-x|=|g(s)-g(x)|=\left|\psi^{-1} g(s)-\psi^{-1} g(x)\right|=\left|s-\psi^{-1} g(x)\right| .
$$

Hence $S$ is covered by the perpendicular bisector of $x$ and $\psi^{-1} g(x)$; therefore $S$ is collinear, which is a contradiction.

Now we prove that $f$ is a local isometry. Let $z \in U$ be arbitrary. Then there exists $r>0$ such that $B(z, 2 r) \subseteq U$. We prove that $f$ is an isometry on $B(z, r)$. 
As $f$ is 1-Lipschitz, we have $|f(x)-f(y)| \leq|x-y|$ for all $x, y \in U$. Assume to the contrary that there are $x, y \in B(z, r)$ such that $|f(x)-f(y)|<|x-y|$. Consider

$$
C_{x, y}=B(x,|x-y| / 2) \cup B(y,|x-y| / 2) .
$$

Clearly, $C_{x, y} \subseteq B(z, 2 r) \subseteq U$. Since $f$ is 1-Lipschitz, $f\left(C_{x, y}\right)$ is contained in the union of two balls $B(f(x),|x-y| / 2) \cup B(f(y),|x-y| / 2)$. Since $|f(x)-f(y)|<|x-y|$, the area of this union is smaller than the area of $C_{x, y}$; thus $\lambda\left(f\left(C_{x, y}\right)\right)<\lambda\left(C_{x, y}\right)$. Applying that $\lambda$ is subadditive and $f$ is 1-Lipschitz we obtain

$$
\lambda(f(U)) \leq \lambda\left(f\left(C_{x, y}\right)\right)+\lambda\left(f\left(U \backslash C_{x, y}\right)\right)<\lambda\left(C_{x, y}\right)+\lambda\left(U \backslash C_{x, y}\right)=\lambda(U),
$$

which is a contradiction.

Since $f$ is a local isometry, it is locally a restriction of unique affine maps. As $U$ is open and connected, $f$ is the restriction of a unique affine map $\psi: \mathbb{R}^{2} \rightarrow \mathbb{R}^{2}$, and clearly $\psi$ is an isometry.

Now we are ready to prove Theorem 1.5 .

Proof of Theorem 1.5. Let $B$ be the closed unit ball centered at the origin and let $C \subseteq[0,1]$ be a nowhere dense compact set with positive one-dimensional Lebesgue measure. Set $U=\operatorname{int}(B) \backslash(C \times[0,1])$. Clearly, $U$ is open and path-connected. It is easy to see that every simple closed curve can be shrunk to a point continuously in $U$, so $U$ is simply connected. Clearly, cl $U=B$ and $\lambda(U)<\lambda(B)$.

It is enough to prove that there is an $\varepsilon>0$ such that if $f: U \rightarrow \mathbb{R}^{2}$ is a contraction with $\lambda(f(U)) \geq \lambda(U)-\varepsilon$, then $\lambda(\partial f(U))>0$. Assume to the contrary that for all $n \in \mathbb{N}^{+}$there are contractions $g_{n}: U \rightarrow \mathbb{R}^{2}$ such that $\lambda\left(g_{n}(U)\right) \geq \lambda(U)-1 / n$ and $\lambda\left(\partial g_{n}(U)\right)=0$. Clearly, we may assume that $\bigcup_{n=1}^{\infty} g_{n}(U)$ is bounded. Let $\left\{z_{i}: i \in \mathbb{N}\right\}$ be a dense set in $U$. By Cantor's diagonal argument we can choose a strictly increasing subsequence of the positive integers $\left\langle n_{k}\right\rangle$ such that for every $i \in \mathbb{N}$ the $\operatorname{limit} \lim _{k \rightarrow \infty} g_{n_{k}}\left(z_{i}\right)$ exists. Since the maps $g_{n_{k}}$ are contractions, the sequence of functions $\left\langle g_{n_{k}}\right\rangle$ is uniformly convergent on $U$. Hence by passing to this subsequence and renumbering it we may assume that $g_{n}$ converges uniformly to a map $g: U \rightarrow \mathbb{R}^{2}$. The uniform convergence implies that $g$ is 1-Lipschitz.

First we prove that $\lambda(g(U))=\lambda(U)$. Since $g$ is 1-Lipschitz, $\lambda(g(U)) \leq \lambda(U)$, so it is enough to prove the opposite direction. As a continuous image of an open set, $g(U)$ is $\sigma$-compact and so measurable. Let $\delta>0$ be arbitrary. The regularity of the Lebesgue measure implies that there is an open set $V$ such that $g(U) \subseteq V$ and $\lambda(V)<\lambda(g(U))+\delta$. Similarly, there exists a compact set $K$ such that $K \subseteq U$ and $\lambda(U \backslash K)<\delta$. Since the maps $g_{n}$ are contractions, we obtain for all $n \in \mathbb{N}^{+}$

$$
\lambda\left(g_{n}(U)\right)-\lambda\left(g_{n}(K)\right) \leq \lambda\left(g_{n}(U \backslash K)\right) \leq \lambda(U \backslash K)<\delta .
$$

The uniform convergence $g_{n} \rightarrow g$ yields that there is an integer $L$ such that for all $n>L$ we have $g_{n}(K) \subseteq V$. Therefore (1) and the definition of the maps $g_{n}$ imply that for all $n>L$ we obtain

$$
\lambda(g(U))+\delta>\lambda(V) \geq \lambda\left(g_{n}(K)\right)>\lambda\left(g_{n}(U)\right)-\delta \geq \lambda(U)-1 / n-\delta .
$$

As $\delta>0$ is arbitrary, we obtain $\lambda(g(U)) \geq \lambda(U)$, so $\lambda(g(U))=\lambda(U)$. Then Lemma 3.1 implies that $g$ is the restriction of an (affine) isometry $\psi: \mathbb{R}^{2} \rightarrow \mathbb{R}^{2}$. By replacing $g_{n}$ with $\psi^{-1} \circ g_{n}$ we may assume that $g$ is the identity, that is, $g=\mathrm{id}_{U}$. 
Since $\operatorname{cl} U=B$, one can extend the maps $g_{n}$ to contractions $\widehat{g}_{n}: B \rightarrow \mathbb{R}^{2}$. Clearly, $\widehat{g}_{n} \rightarrow \mathrm{id}_{B}$ uniformly on $B$. Let $D \subseteq B$ be a closed ball centered at the origin such that $\lambda(U)<\lambda(D)<\lambda(B)$. There exists $M \in \mathbb{N}$ such that for all $n>M$ we have

$$
\max _{x \in B}\left|\widehat{g}_{n}(x)-x\right|<\operatorname{dist}(D, \partial B) .
$$

We prove for all $n>M$ that

$$
D \subseteq \operatorname{cl}\left(g_{n}(U)\right) .
$$

Let us fix $n>M$. As $g_{n}(U)$ is dense in $\widehat{g}_{n}(B)$, we obtain $\operatorname{cl}\left(g_{n}(U)\right)=\widehat{g}_{n}(B)$. Thus we need to prove $D \subseteq \widehat{g}_{n}(B)$ for (3). Assume to the contrary that there is an $x_{0} \in D$ such that $x_{0} \notin \widehat{g}_{n}(B)$. Set $r=\operatorname{dist}(D, \partial B)$. Then $B\left(x_{0}, r\right) \subseteq B$, so we can define the map $\phi: B\left(x_{0}, r\right) \rightarrow \mathbb{R}^{2}$ by $\phi(x)=-\widehat{g}_{n}(x)+x+x_{0}$. Inequality (2) implies $\left|\phi(x)-x_{0}\right|<r$, so $\phi\left(B\left(x_{0}, r\right)\right) \subseteq B\left(x_{0}, r\right)$. Since $x_{0} \notin \widehat{g}_{n}(B)$, we obtain that $\phi(x) \neq x$ for all $x \in B\left(x_{0}, r\right)$. Hence $\phi$ is a continuous self-map of the ball $B\left(x_{0}, r\right)$ without any fixed points, which contradicts the Brouwer Fixed Point Theorem [3, Proposition 4.4.]. Thus (3) holds.

As the maps $g_{n}$ are contractions, $\lambda\left(g_{n}(U)\right) \leq \lambda(U)$. Therefore $\lambda(U)<\lambda(D)$ and (3) imply, for all $n>M$, that

$$
\begin{aligned}
\lambda\left(\partial g_{n}(U)\right) & \geq \lambda\left(\operatorname{cl}\left(g_{n}(U)\right) \backslash g_{n}(U)\right) \\
& \geq \lambda\left(\operatorname{cl}\left(g_{n}(U)\right)\right)-\lambda\left(g_{n}(U)\right) \\
& \geq \lambda(D)-\lambda(U)>0 .
\end{aligned}
$$

Thus $\lambda\left(\partial g_{n}(U)\right)>0$, which contradicts the definition of $g_{n}$. This concludes the proof.

Remark 3.2. It is easy to see that for all Lebesgue null sets $N \subseteq \mathbb{R}^{2}$ the sets $U \Delta N$ are also counterexamples to Question 1.4. On the other hand, one can show that for all $\varepsilon>0$ there exist a contraction $f: U \rightarrow \mathbb{R}^{2}$ and a Lebesgue null set $N \subseteq \mathbb{R}^{2}$ such that $\lambda^{2}(f(U)) \geq \lambda^{2}(U)-\varepsilon$ and $f(U) \Delta N$ is a polygon. Thus $U$ will not be a counterexample to Question 4.4 below.

\section{Open questions}

The most important question is the following.

Question 4.1. Let $K \subseteq \mathbb{R}^{2}$ be a compact set, and let $\varepsilon>0$. Does there exist a contraction $f: K \rightarrow \mathbb{R}^{2}$ such that $\lambda^{2}(f(K)) \geq \lambda^{2}(K)-\varepsilon$ and $f(K)$ is a polygon?

In order to answer Question 4.1 we consider the next question.

Question 4.2. Let $C \subseteq \mathbb{R}^{2}$ be a compact set with $\lambda^{2}(C)=0$, and let $\varepsilon>0$. Does there exist a contraction $f: C \rightarrow \mathbb{R}^{2}$ such that $|f(x)-x| \leq \varepsilon$ for all $x \in C$ and $f(C)$ can be covered by finitely many line segments?

If the compact set $C$ is a counterexample to Question 4.2 with $\varepsilon>0$, then consider $K=C \cup R$, where $R$ is a closed ring such that the bounded component of its complement contains $C$. Then $K$ is a counterexample to Question 4.1, the sketch of the proof is the following. Assume to the contrary that there are contractions $f_{n}: K \rightarrow \mathbb{R}^{2}\left(n \in \mathbb{N}^{+}\right)$such that $\lambda\left(f_{n}(K)\right) \geq \lambda(K)-1 / n$ and $f_{n}(K)$ is a polygon; that is, $\partial f_{n}(K)$ can be covered by finitely many line segments. Similarly as in the proof of Theorem 1.5. one can show that $f_{n}$ converges uniformly to an isometry, $f$. We may assume that $f=\operatorname{id}_{K}$. Let us fix $n \in \mathbb{N}^{+}$such that $\left|f_{n}(x)-x\right| \leq \varepsilon$ 
for all $x \in C$ and $f_{n}(C) \cap f_{n}(R)=\emptyset$. As $f_{n}$ is a contraction and $C$ has zero Lebesgue measure, $f_{n}(C)$ also has zero measure, so $\operatorname{dist}\left(f_{n}(C), f_{n}(R)\right)>0$ implies $f_{n}(C) \subseteq \partial f_{n}(K)$. Therefore $f_{n}(C)$ can be covered by finitely many line segments, which contradicts the choice of $C$ and $\varepsilon$.

Remark 4.3. We do not know whether the Sierpiński carpet is a counterexample to Question 4.2 ,

Finally, our last question is the following.

Question 4.4. Let $E \subseteq \mathbb{R}^{2}$ be a measurable set with $\lambda^{2}(E)<\infty$, and let $\varepsilon>0$. Do there exist a contraction $f: E \rightarrow \mathbb{R}^{2}$ and a Lebesgue null set $N \subseteq \mathbb{R}^{2}$ such that $\lambda^{2}(f(E)) \geq \lambda^{2}(E)-\varepsilon$ and $f(E) \Delta N$ is a polygon? Is this true at least for compact sets?

\section{ACKNOWLEDGEMENTS}

The authors are indebted to M. Laczkovich for some valuable remarks and helpful suggestions.

\section{REFERENCES}

[1] P. Alexandroff, letter to Felix Hausdorff, 27 December 1932.

[2] Jürgen Elstrodt, Alte Briefe-aktuelle Fragen aus Hausdorffs Briefwechsel (German), Mitt. Dtsch. Math.-Ver. 18 (2010), no. 3, 183-187. MR2761783

[3] William Fulton, Algebraic topology, Graduate Texts in Mathematics, vol. 153, Springer-Verlag, New York, 1995. A first course. MR1343250 (97b:55001)

[4] Miklós Laczkovich, Paradoxical decompositions using Lipschitz functions, Real Anal. Exchange 17 (1991/92), no. 1, 439-444. MR1147388 (93f:28008)

[5] Jiří Matoušek, On Lipschitz mappings onto a square, The mathematics of Paul Erdős, II, Algorithms Combin., vol. 14, Springer, Berlin, 1997, pp. 303-309, DOI 10.1007/978-3-64260406-5_27. MR.1425223 (98g:28009)

[6] Nguyen Xuan Uy, Removable sets of analytic functions satisfying a Lipschitz condition, Ark. Mat. 17 (1979), no. 1, 19-27, DOI 10.1007/BF02385454. MR543500 (81i:30076)

Alfréd Rényi Institute of Mathematics, Hungarian Academy of Sciences, P.O. Box 127, 1364 Budapest, Hungary

E-mail address: balka.richard@renyi.mta.hu

Current address: Department of Mathematics, University of Washington, Box 354350, Seattle, Washington 98195-4350

Alfréd Rényi Institute of Mathematics, Hungarian Academy of Sciences, P.O. Box 127, 1364 Budapest, Hungary - And - Institute of Mathematics, Eötvös Loránd UniverSity, PÁzmány PÉter s. 1/C, 1117 Budapest, Hungary

E-mail address: elekes.marton@renyi.mta.hu

Mathematics Institute, University of Warwick, Coventry, CV4 7AL, United Kingdom E-mail address: A.Mathe@warwick.ac.uk 